Original Papers

\title{
Development of Directly Coupled Supercritical Fluid Chromatography-Mass Spectrometry with Self-Spouting and Vacuum Nebulizing Assisted Interface
}

\author{
Kozo Matsumoto*, Shin Tsuge* and Yukio Hirata** \\ *Department of Synthetic Chemistry, Faculty of Engineering, Nagoya University, \\ Nagoya 464 \\ **School of Materials Science, Toyohashi University of Technology, Toyohashi 440
}

\begin{abstract}
Supercritical fluid chromatograph $(\mathrm{SFC})$ with a fused silica separation column $(0.5 \mathrm{~mm} 1 . D . \times 70 \mathrm{~cm})$ packed with ODS-Q3 $(30-50 \mu \mathrm{m})$ was directly coupled with a quadrupole mass spectrometer (MS) through a vacuum nebulizing interface originally developed for the combination of high performance liquid chromathograph (HPLC) with MS. Mixtures of hexane and ethanol were used as the mobile phase for SFC at a flow rate of $40 \mu \mathrm{l} / \mathrm{min}\left(50 \mathrm{~kg} / \mathrm{cm}^{2}\right)$. The column temperatures were maintained above the critical ones of the mobile phase between 260 and $280^{\circ} \mathrm{C}$. Introduction of line filters to remove possible small particles in the SFC effluent and the subtle control of the optimum flow and/or the pressure for the SFC column enabled fairly long term stable operation for this SFC-MS system. Thus developed new system was successfully applied to the analysis of various samples such as styrene oligomers, phthalates, triglycerides, and polyethylene glycol derivatives.
\end{abstract}

Keywords Supercritical fluid chromatography (SFC), vacuum nebulizing interface, mass spectrometry

Supercritical fluid chromatography(SFC) has been vigorously investigated and recognized as a rapid and powerful technique for separating non-volatile and thermally unstable compounds. SFC has various specific features concerning the larger diffusion coefficient and the smaller viscosity of the associated mobile phases comparing with conventional high performance liquid chromatography (HPLC). The solvation power of the mobile phase to influence the separation can easily be changed over a wide range simply by adjusting the pressure and the temperature. In addition to this, SFC is principally much feasible to make a direct coupling system with a mass spectrometer (SFC-MS) since most of the mobile phases for SFC such as carbon dioxide and pentane have much higher volatility than those for ordinary HPLC. ${ }^{1-4}$

Randall et al. ${ }^{1}$ first reported on the construction of SFC-MS interface by using packed columns and supersonic molecular beam technique. The mass spectra of anthracene $(\mathrm{MW}=178)$ and 1,3,5-triphenylbenzene $(\mathrm{MW}=306)$ were reported. On the other hand, Smith et al..$^{2-4}$ developed an interface for open tubular capillary column SFC-MS. They used long fused silica open tubular capillaries (typically 50-100 $\mu \mathrm{m}$ I.D. $\times$ $10-30 \mathrm{~m}$ ) as the separation columns. They successfully applied the system to various non-volatile and/or thermally labile compounds such as polycyclic aromatic hydrocarbons, polystyren oligomers, mycotoxins, and marine pollutants. However, the selection of the stationary phases were restricted mostly to those crosslinked and/or chemically bonded to the inner wall of the open tubular capillary column. In addition, in this case the end of the capillary coulmn has to be interfaced either by a diaphram with a $0.5-2.0 \mu \mathrm{m}$ pinhole, a pinched Pt-Ir tubing or a piece of narrowbore $(5 \mu \mathrm{m}$ I.D.) fused sillica capillary to provide the appropriate back pressure for the SFC column. These narrow orifices, however, are often plugged by small particles in the effluent. Furthermore, the column conditions for SFC are susceptible to change depending on the resistance of the interface.

One of the authors has been working on SFC with fused silica packed colulmns $(0.2 \mathrm{~mm}$ I.D. $\times 50-70$ $\mathrm{cm}){ }^{5-7}$ Pure haxane and ethanol-doped hexane were used as the mobile phase at the pressures between $40-70$ atm and the temperatures between $250-280^{\circ} \mathrm{C}$. By use of small bore fused silica packed columns, the SFC system could be simplified without sacrificing the highresolution mainly because of the rapid heat exchange through the thin wall of the capillary column and the 
small pressure drop of the short packed column. Furthermore, in this case any types of packing materials developed for the conventional HPLC are directly adopted to the SFC columns, and relatively large sample loading is acceptable.

In this work, the SFC with the fused silica packed column mentioned above was directly couppled with a quadrupole MS through the vacuum nebulizing interface originally developed for HPLC-MS. Here, a relatively larger bore capillary $(0.5 \mathrm{~mm} \mathrm{I.D.} \times 70 \mathrm{~cm})$ was used to facilitate the introduction of line filters (1 $\mu \mathrm{m}$ pore size) to remove possible small particles in the effluent without band broadening. By this, considerably long term stability of the nebulization was attained. Furthermore, by this interface the optimum flow and/or the pressure control of the SFC column were easily attained by adjusting the splitting vent system incorporated in the nebulizing coaxial nozzle. The separated components by SFC were monitored successively by UV and MS. Thus developed SFC-MS system was successfully applied to various samples such as polystyrene oligomers, phthalates, triglycerides, and polyethylene glycol (PEG) derivatives.

\section{Experimental}

\section{Samples and reagents}

Polystyrene oligomers of A-500 were purchased from Toyo Soda Manufacturing Co., Ltd.; triglycerides of trilaurin, tri- $n$-caprin, and tri- $n$-caprylin from Wako Chemical Industries, Ltd.; phthalates such as lauryl-, dioctyl-, diisobutyl-, diisopropyl-, and dimethylphthalates, and polyethylen-glycol-mono- $p$-nonyl-phenyl-ether and polyethylene-glycol-mono-oleyl-ether from Tokyo Kasei. These samples were used without further purification. Ethanol and hexane were of HPLC grade from Wako Chemical Industries, Ltd.

\section{$S F C-M S$}

A schematic diagram of the SFC-MS system used for this work is shown in Fig. 1. The SFC apparatus comprizes a Shimadzu LC-5A pump to generate high pressure for the mobile phase, and a Hitachi K-53 gas chromatograph oven to provide constant temperature conditions. Mixtures of hexane and ethanol were used as the mobile phase. The samples were dissolved in hexane at $1 \mathrm{mg} / \mathrm{ml}$. A degasser (Erma Optical Works, LTD.) was used to remove air dissolved in the mobile phase. The sample solutions and the mobile phases were filtrated through a $0.5 \mu \mathrm{m}$ filter (Millex-SR) to remove small particles. The pump was operated under constant pressure mode at $50 \mathrm{~kg} / \mathrm{cm}^{2}$ at a flow rate of $40 \mu \mathrm{l} / \mathrm{min}$. Sample injection was accomplished using a $1 \mu \mathrm{l}$ valve injector (JASCO) mounted exterior of the oven. A fused silica capillary tubing ( $0.5 \mathrm{~mm}$ I.D. $\times 70$ $\mathrm{cm})$ packed with ODS-Q3 of 30-50 $\mu \mathrm{m}$ was used as the separation column. The column was maintained at a constant temperature above the critical temperature of the mobile phase in the gas chromatograph oven between 260 and $280^{\circ} \mathrm{C}$. The supercritical effulent was cooled down to the liquid before the UV detector in order to make a serial monitoring by UV and MS.

The outlet of the UV detector was directly connected to the coaxial capillary tubing of the vacuum nebulizing interface originally developed for HPLC-MS coupling ${ }^{8}$ by a stainless steel capillary tubing $(0.13 \mathrm{~mm}$ I.D. $\times 30$ $\mathrm{cm}$ ) of which dead volume was ca. $4 \mu \mathrm{l}$. Two line filters $(1 \mu \mathrm{m}$ pore size) were incorporated at the both ends of the stainless connecting tube to protect the nebulizing nozzle from dust. The coaxial capillary tubing for the nebulizing interface, which is basically the same as in the previous HPLC-MS work ${ }^{8}$, consists of an outer stainless tube $(0.63 \mathrm{~mm}$ O.D. $\times 0.33 \mathrm{~mm}$ I.D.) and an inner stainless tube (0.31 mm O.D. $\times 0.13 \mathrm{~mm}$ I.D.) to enable flow and pressure control through a splitting vent. At the nebulizing tip, a piece of glass capillary (0.12 mm O.D. $\times 0.012 \mathrm{~mm}$ I.D.) is fixed to attain self-spouting of the effluent. Once the whole SFC effluent carried to just before the nebulizing tip through the inner tube, one portion of the effluent is introduced into the self-spouting capillary nozzle, and the rest of the effluent is carried back through the gap between the outer and the inner tubes to the splitting

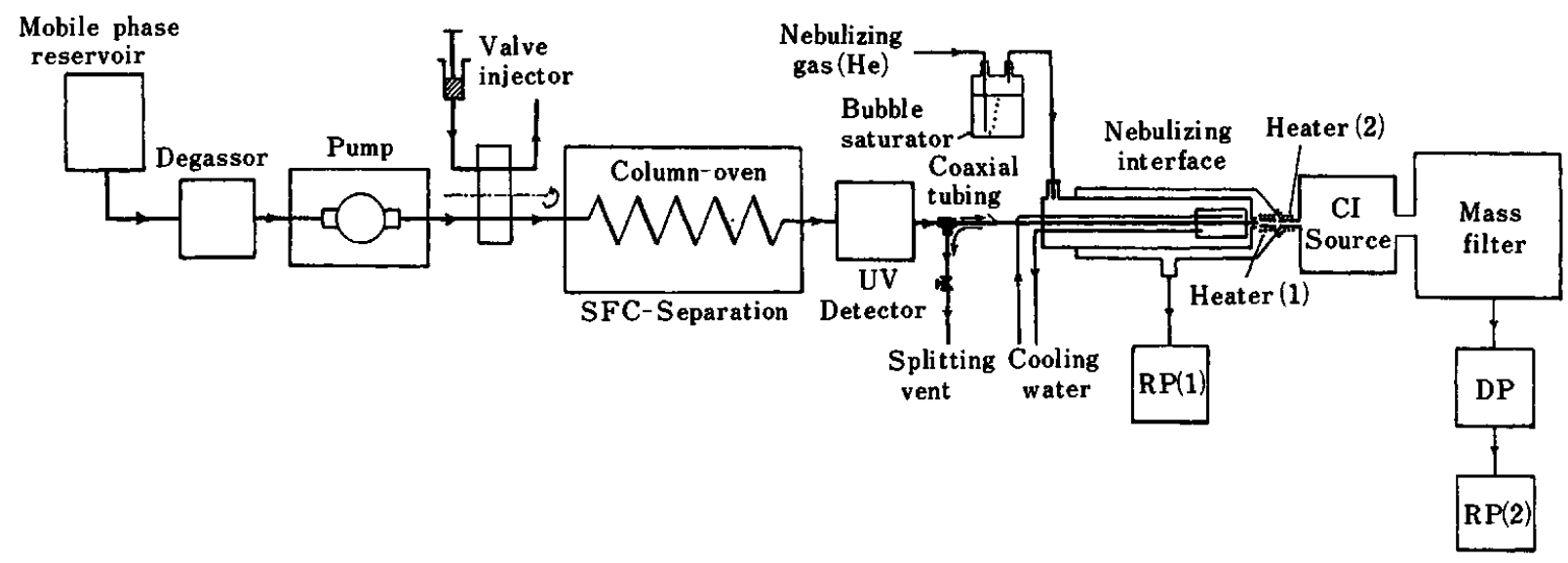

Fig. I Schematic diagram of SFC/MS combination system. 
vent. The spouting height (amounts) of the effluent can be controlled by adjusting the needle valve before the vent. Ordinally about half of the whole effluent (ca. $20 \mu \mathrm{l} / \mathrm{min}$ ) was vented.

The nebulizing gas (He) saturated with the vapor of methanol was used to attain stable nebulization ${ }^{9}$ and modify the CI reagent (mostly the SFC-solvent vapors) with methanol. The nebulized effluent is then desolvated by the aid of the electric heating between the nebulizing tip and the counter skimmer. Ordinally the surface temperature of the desolvation Heater( 1 ) and that of the maintenance Heater(2) were kept at ca. 700 and $200^{\circ} \mathrm{C}$, respectively. Most of the solvent vapors are evacuated off through the RP(1). Thus formed sample vapor as well as some of the solvent vapor are introduced through the skimmer into the chemical ionization ( $\mathrm{CI}$ ) source of a quadrupole $\mathrm{MS}, \mathrm{Q}-10 \mathrm{~A}$ (JEOL). Here, the vapors of the mobile phase plus methanol from the nebulizing gas were used as the reagent gas for $\mathrm{CI}$. The temperature of the $\mathrm{CI}$-source was maintained at $c a .180^{\circ} \mathrm{C}$. The MS was ordinally scanned from 150 to 550 amu (the maximum of this instrument utilized) at $3 \mathrm{~s}$ per scan.

\section{Results and Discussion}

Figure 2 shows the UV trace at $210 \mathrm{~nm}$ and those of selected ion monitoring (SIM) for a polystyrene

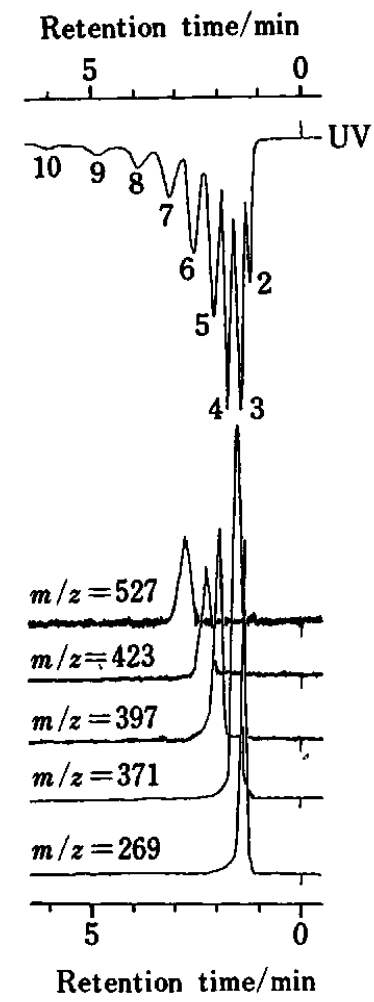

Fig. 2 Chromatograms of a polystyrene oligomer sample by UV and SIM, the number on the peaks of the UV trace are those of $n$ for $\left.\mathrm{C}_{4} \mathrm{H}_{9}+\mathrm{CH}_{2}-\mathrm{CHPh}\right)_{n} \mathrm{H}$. oligomer sample, A-500, $\mathrm{C}_{4} \mathrm{H}_{9}+\mathrm{CH}_{2}-\mathrm{CHPh}$ $_{n} \mathrm{H}$, (the mean $n=5$ ). In this case, the column temperature was maintained at $260^{\circ} \mathrm{C}$ for a mobile phase of a mixture (hexane:ethanol $=9: 1$ ). The corresponding high quality CI mass spectrum was also successively obtained for each peak on the UV trace with a time lag of $c a .20 \mathrm{~s}$. Figure 3 shows the typical mass spectrum for the trimer $(n=3)$ peak of which retention time $\left(R_{1}\right)$ is ca. $1.3 \mathrm{~min}$ on the UV trace. In this case, [MH$\left.2 \mathrm{C}_{6} \mathrm{H}_{6}\right]^{+}(m / z=215),\left[\mathrm{MH}-\mathrm{C}_{6} \mathrm{H}_{6}\right]^{+} \quad(m / z=293)$, and $[\mathrm{MH}]^{+}(m / z=371)$, are observed in the relative intensities of 100,61 , and 35 , respectively. On the mass spectrum of the tetramer $(n=4),[\mathrm{MH}]^{+}(m / z=475)$ was no more observable, and instead $\left[\mathrm{MH}-2 \mathrm{C}_{6} \mathrm{H}_{6}\right]^{+}(m /$ $z=397)$ and $\left[\mathrm{MH}-\mathrm{C}_{6} \mathrm{H}_{6}\right]^{+}(m / z=319)$ were observed in the relative intensities of 100 and 24 respectively. For

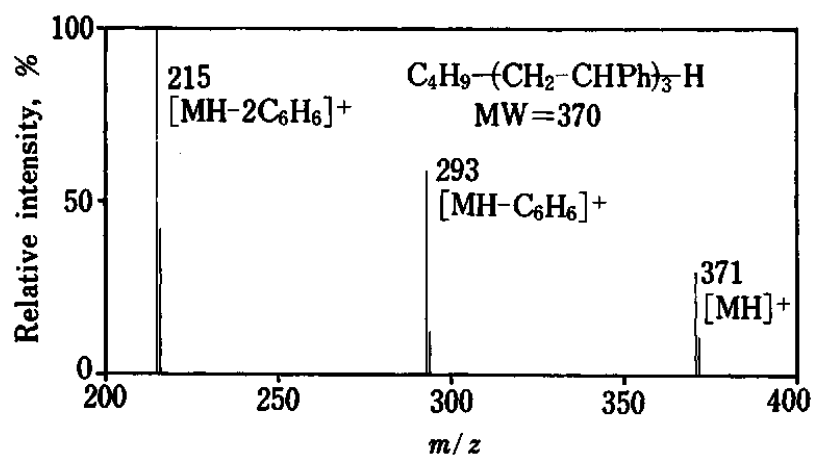

Fig. 3 Cl mass spectrum of the styrene trimer.

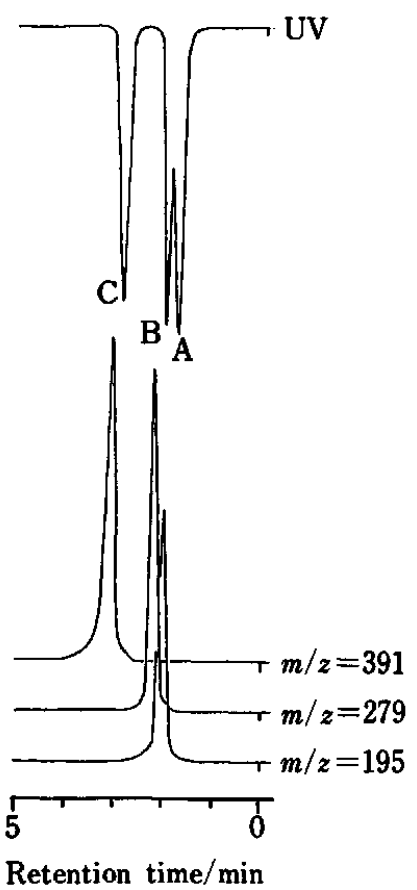

Fig. 4 Chromatograms of a mixture of dimethyl(A)-, diisobutyl(B)-, and dioctyl(C)- phthalates by UV and SIM. 
the oligomers $(n=5$ and 6$)$ only $\left[\mathrm{MH}-2 \mathrm{C}_{6} \mathrm{H}_{6}\right]^{+}$were observed at $m / z=423$ and 527 , respectively. The highest mass number of each mass spectrum was monitored for the SIM in Fig. 2. Although on the UV trace up to 10 mer $(n=10)$ were observed, only the mass fragmentograms up to 6 mer were monitored because of the mass range limitation of the instrument utilized. The observed SIM traces also suggest fairly stable sample introduction for the SFC effluent by the nebulizing interface.

Figure 4 shows the chromatograms of a mixture of dimetyl(A)-, diisobutyl(B)- and $\operatorname{dioctyl}(\mathrm{C})$-phthalates detected by UV at $210 \mathrm{~nm}$, and SIM at $m / z=195,279$, and 391, respectively. The SFC conditions are the same as in the polystyren oligomers. Figure 5 shows the CI mass spectra for the three components. Although the separation of dimethyl phthalate (A) and diisobutyl phthalate (B) is not complete on the UV trace under the given conditions, the SIM provides perfect discrimination of the two components. Figure 6 shows the UV and SIM traces of a crude laulyl phthalate sample. This sample contains many components, which can be easily assigned from their retention times $\left(R_{1}\right)$ and mass spectra. The middle three peaks (E, $F$ and $G$ ), for example, are assigned from their mass spectroscopic data as phthalates such as $\mathrm{C}_{6} \mathrm{H}_{4}\left(\mathrm{COOC}_{12} \mathrm{H}_{25}\right)\left(\mathrm{COOC}_{10} \mathrm{H}_{21}\right)(\mathrm{Cl2}: \mathrm{Cl0}, \mathrm{MW}=$ 474), $\mathrm{C}_{6} \mathrm{H}_{4}\left(\mathrm{COOC}_{12} \mathrm{H}_{25}\right)\left(\mathrm{COOC}_{12} \mathrm{H}_{25}\right)(\mathrm{Cl2}: \mathrm{Cl} 2, \mathrm{MW}=$ 502), and $\mathrm{C}_{6} \mathrm{H}_{4}\left(\mathrm{COOC}_{12} \mathrm{H}_{25}\right)\left(\mathrm{COOC}_{14} \mathrm{H}_{29}\right)(\mathrm{Cl2}: \mathrm{Cl4}$, $\mathrm{MW}=530$ ), respectively. Combining the linear relationship between the total carbon number of two ester groups $\left(C_{x}+C_{y}\right)$ and the logalism of the retention time of the phthatalte with the mass spectroscopic data, the peak assignment was unequivocally made for the phthalates on the chromatogram by SFC; $\mathrm{A}(\mathrm{C6}$ : $\mathrm{C} 6)$,

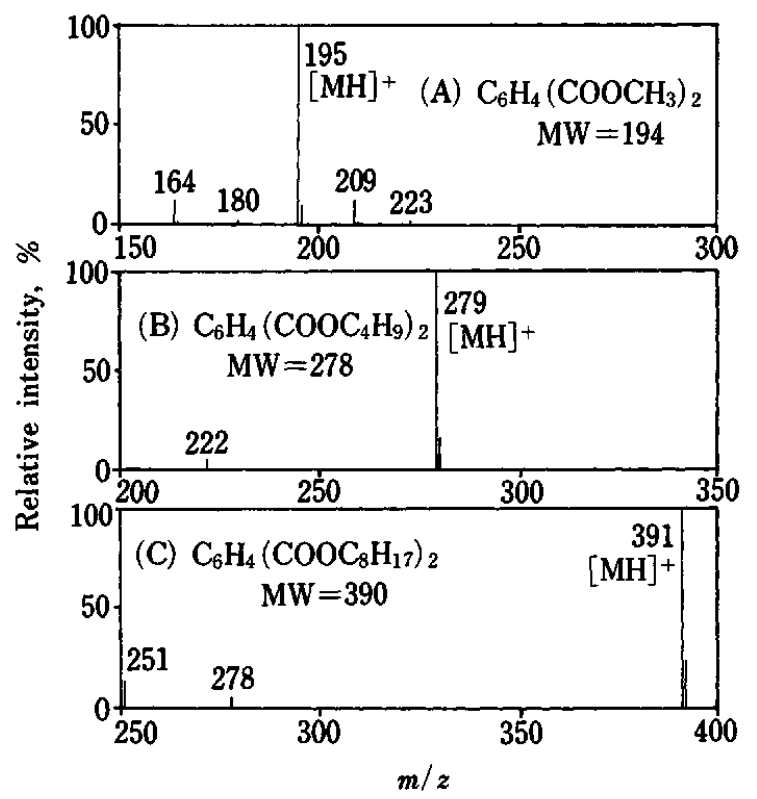

Fig. 5 CI mass spectra of dimethyl(A)- diisobutyl(B)-, and dioctyl(C)- phthalates.
$\mathrm{B}(\mathrm{C} 8: \mathrm{C} 8), \quad \mathrm{C}(\mathrm{C} 8: \mathrm{Cl0}), \quad \mathrm{D}(\mathrm{Cl0}: \mathrm{Cl0}), \quad \mathrm{E}(\mathrm{Cl0}: \mathrm{Cl} 2)$, $\mathrm{F}(\mathrm{Cl2}: \mathrm{Cl} 2), \mathrm{G}(\mathrm{Cl2}: \mathrm{Cl} 4), \mathrm{H}(\mathrm{Cl}: \mathrm{Cl} 14), \mathrm{I}(\mathrm{Cl} 4: \mathrm{Cl})$, $\mathrm{J}(\mathrm{Cl6}$ : $\mathrm{Cl6})$ and $\mathrm{K}(\mathrm{Cl6}$ : $\mathrm{C} 18)$, respectively.

Figure 7 shows the UV and SIM traces of a mixture of three crude triglycerides of tri- $n$-caprylin(A), tri- $n$ caprin(B), and trilaurin(C). The SFC conditions are the same as in the polystyrene oligomer analysis. SIM's for tri- $n$-caprylin, tri- $n$-caprin and tri- $n$-laurin were made at the base peaks such as [MH-

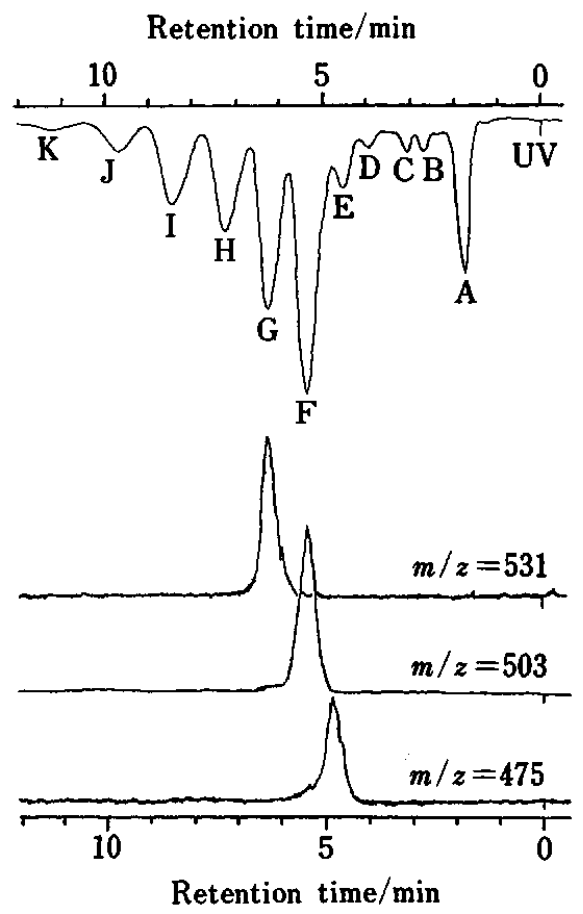

Fig. 6 Chromatograms of a crude lauryl phthalate sample by UV and SIM.

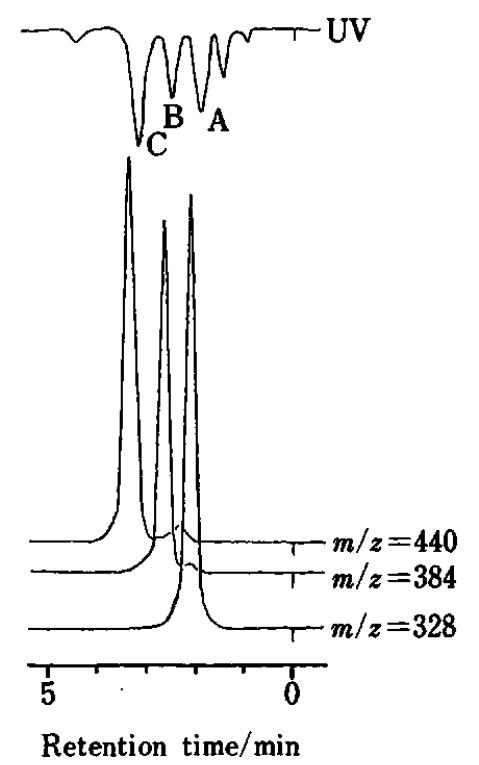

Fig. 7 Chromatograms of a mixture of three triglycerides, tri- $n$-caprylin, tri- $n$-caprin, and trilaurin by $\dot{U V}$ and SIM. 


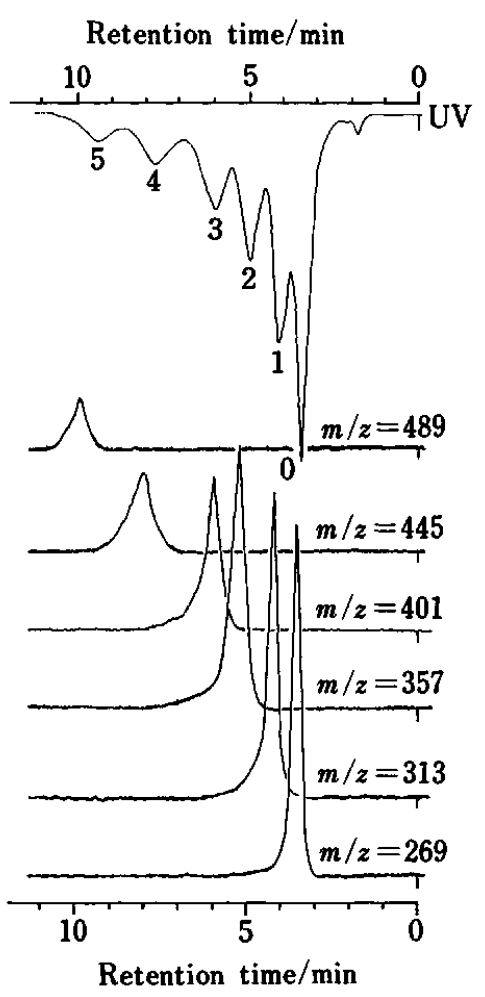

Fig. 8 Chromatograms of a polyethylene-glycol-mono-oleylether sample by UV and SIM, the number on the peaks of the UV trace are those of $n$ for $\mathrm{C}_{18} \mathrm{H}_{35}+\mathrm{OCH}_{2} \mathrm{CH}_{2}{ }_{n} \mathrm{OH}$.

$\left.\mathrm{C}_{7} \mathrm{H}_{15} \mathrm{COO}\right]^{+}$at $m / z=328,\left[\mathrm{MH}-\mathrm{C}_{9} \mathrm{H}_{19} \mathrm{COO}^{+}\right.$at $\mathrm{m} /$ $z=384$ and $\left[\mathrm{MH}-\mathrm{C}_{11} \mathrm{H}_{23} \mathrm{COO}\right]^{+}$at $m / z=440$, respectively. Although, triglycerides are less sensitive on UV at $200 \mathrm{~nm}$, the SIM are very sensitive.

Finally, polyethylene-glycol-mono-oleyl-ether, $\left.\mathrm{C}_{18} \mathrm{H}_{35}+\mathrm{OCH}_{2} \mathrm{CH}_{2}\right)_{n} \mathrm{OH}$ (mean $n=2$ ) was separated by a mobile phase of $30 \%$ ethanol in hexane at a temperature of $280^{\circ} \mathrm{C}$. The obtained UV and SIM traces are shown in Fig. 8. The mass spectra of the oligomers $(n=0$ to 5$)$ shown in Fig. 9 mainly consist of the quasi-molecular peaks, $[\mathrm{MH}]^{+}$, and some fragment ones and the SIM's were made at the $[\mathrm{MH}]^{+}$. Basically the same conditions were also successfully applied to the analysis of polyethylene-glycol-mono-p-nonyl-phenyl-oligomers, $\mathrm{C}_{9} \mathrm{H}_{19}-\mathrm{Ph}+\mathrm{OCH}_{2} \mathrm{CH}_{2}$ 斿 $\mathrm{OH}$ (the mean $n=5$ ).

The present work demonstrates the potential ability of the packed capillary column SFC-MS coupled by the nebulizing interface developed for the combination of HPLC-MS. The proposed system enabled fairly long term stable operation for more than one month with various test samples. Various practical applications of this SFC-MS system are currently in progress.

\section{References}

1. L. G. Randall and A. L. Wahrhaftig, Anal. Chem., 50, 1703 (1978).

2. R. D. Smith, J. C. Fjeldsted and M. L. Lee, $J$. Chromatogr., 247, 231 (1982).
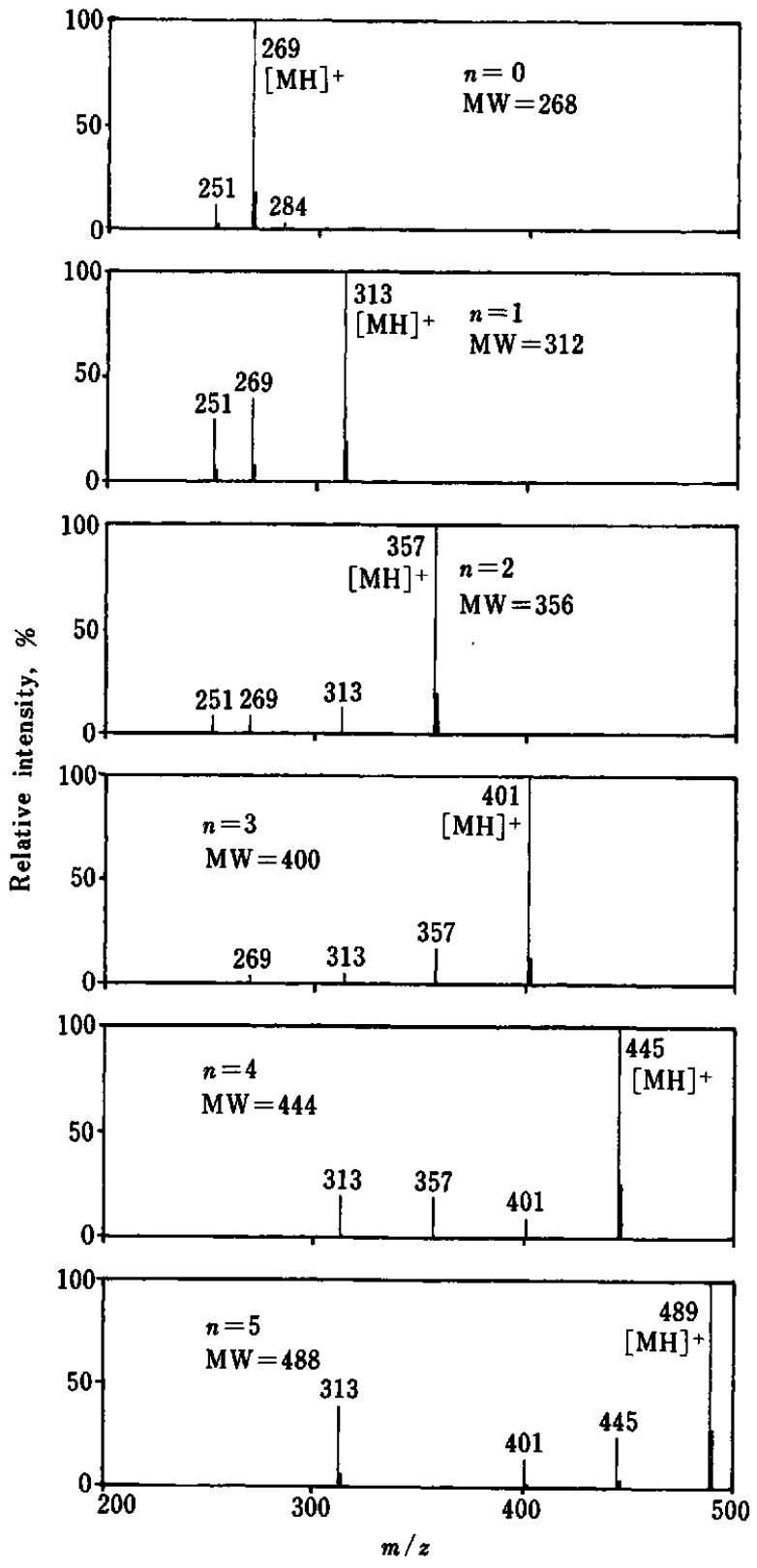

Fig. 9 CI mass spectra of polyetylene-glycol-mono-oleylethers $(n=0$ to 5 ).

3. R. D. Smith and H. R. Udseth, Anal. Chem., 55, 2266 (1983).

4. B. W. Wright, H. R. Udsth, R. D. Smith and R. N. Hazlett, J. Chromatogr., 314, 253 (1984).

5. Y. Hirata and F. Nakata, J. Chromatogr., 295, 315 (1984).

6. Y. Hirata, J. Chromatogr., 315, 31 (1984).

7. Y. Hirata, J. Chromatogr., 315, 39 (1984).

8. K. Matsumoto and S. Tsuge, Fresenius' Z. Anal. Chem., in press.

9. H. Yoshida, K. Matsumoto, K. Itoh, S. Tsuge, Y. Hirata, K. Mochizuki, N. Kokubun, Y. Yoshida, Fresenius' Z. Anal. Chem., 311, 674 (1982).

(Received October 30, 1985) (Accepted November 27, 1985) 